\title{
DC Current Beat: Wireless and Non-Invasive DC Current Sensing Scheme ${ }^{\dagger}$
}

\author{
Koichiro Ishibashi *, Makoto Serizawa, Ryohei Takitoge, Shohei Ishigaki and Tsuyoshi Ishige \\ Advanced Wireless and Communication Research Center, The University of Electro-Communications, \\ Tokyo 182-8585, Japan; s1313095@edu.cc.uec.ac.jp (M.S.); r.takitoge@uec.ac.jp (R.T.); s-ishigaki@uec.ac.jp (S.I.); \\ t.ishige@uec.ac.jp (T.I.) \\ * Correspondence: ishibashi@ee.uec.ac.jp; Tel.: +81-42-443-5188 \\ + Presented at the Eurosensors 2017 Conference, Paris, France, 3-6 September 2017.
}

Published: 24 September 2017

\begin{abstract}
This paper presents a wireless and Non-invasive DC Current (DCC) sensing scheme as an IoT sensors. A RF module transmits only ID codes to a receiver, and the ID transmissions are called as "DCC Beat". The interval time of DCC Beats depend on the inductance of ferrite clamp which is non-invasively installed at the wire of the DC current to be measured, so that the interval time corresponds to DC Current. The ID data transmission range reaches up to $50 \mathrm{~m}$ with $1.2 \mathrm{~mW}$ operating power using a $2.4 \mathrm{GHz}$ RF module. DC current from 0.2 to $4 \mathrm{~A}$ can be measured within error of $5.7 \%$.
\end{abstract}

Keywords: IoT; DC current sensor; non-invasive; beat sensor

\section{Introduction}

For non-invasive DC current measurement, Hall Sensor with ferrite clamp is often used [1]. We have proposed Power Beat Sensor [2] and Temperature Beat Sensor [3] as energy harvesting wireless sensors for IoT applications. This paper first demonstrates DC Current Beat Sensors, in which wireless and non-invasive DC Current (DCC) sensing can be successfully realized, so that it is used as IoT sensors at such various kinds of applications as HEMS, BEMS, DC motor control, Solar Cell control, charge control on secondary batteries, and smart grid using DC power supply. In the DCC Beat Sensors, only ID signal is transmitted by an RF transmitter, the interval time of the ID signal corresponds to the DC current to be measured.

\section{Structure of the DCC Beat Sensor}

The simple circuit for the DCC beat generations consists of DCDC converter, storage capacitor, Voltage Detector (VD), Low Dropout Regulator (LDO), and MCU and a 2.4 GHz RF module [4], and no ADC is required as shown in Figure 1. As shown in Figure 2, voltage of $1.2 \mathrm{~V}$ is leveled up to higher voltages by a switching circuit with an inductances of $L_{1}$ in the DCDC converter, and the storage capacitor CST is charged up. When Schmitt trigger VD detects the predetermined high value $\mathrm{V}_{\mathrm{H}}$ of $2.6 \mathrm{~V}$, the LDO is turned on and it supplies LDO out of $1.9 \mathrm{~V}$, which corresponds to the supply voltage of the MCU and the RF module. Then ID transmission is carried out by the RF module. After the ID transmission is finished, the LDO is turned off. Then the storage capacitor is charged up again followed by the next ID transmission. We use two inductances, where $\mathrm{L}_{1}$ is fixed value, and $\mathrm{L}_{2}$ is depending on DC current (Io) to be measured.

When the nMOS switch at the DCDC converter is on, the current $\mathrm{I}_{1}$ flows on the constant inductance $\mathrm{L}_{1}$, so that energy stored in the inductance $\mathrm{L}_{1}$ becomes $\mathrm{E}_{1}$ as shown Equation (1). When the nMOS switch turns off, the $\mathrm{E}_{1}$ is transferred to the energy on $\mathrm{L}_{2}$ and the storage capacitor CSт as described in Equation (2). So the storage capacitor is charged up as nMOS is switching by the 100 
$\mathrm{KHz}$ pulses. Interval time $(t i)$ of the ID transmissions becomes short when the inductance $\mathrm{L}_{2}$ decreases, where L2 decreases as DC current to be measured increases.

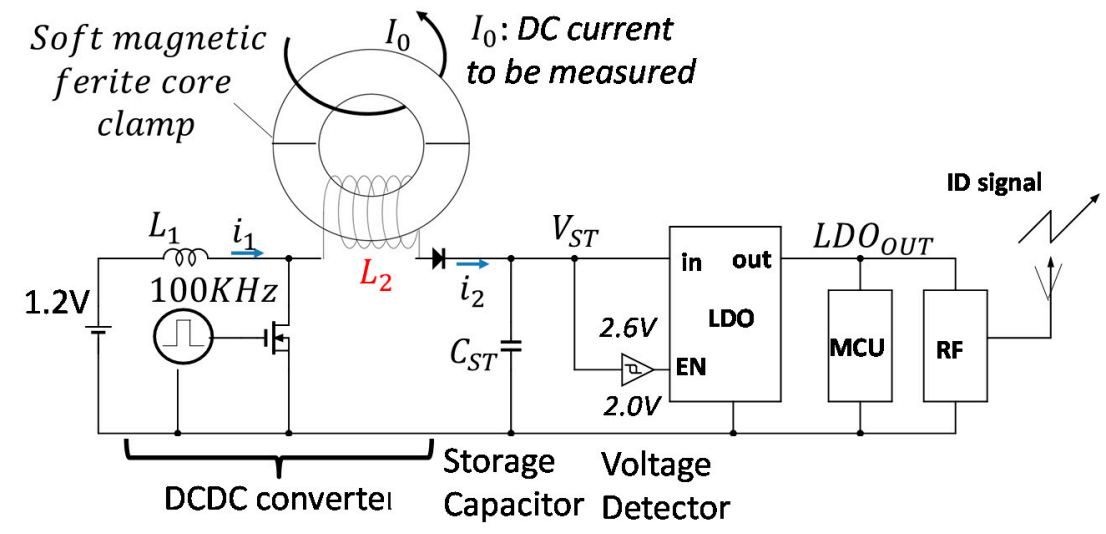

Figure 1. Circuit Structure of the DC Current Beat Sensor node.

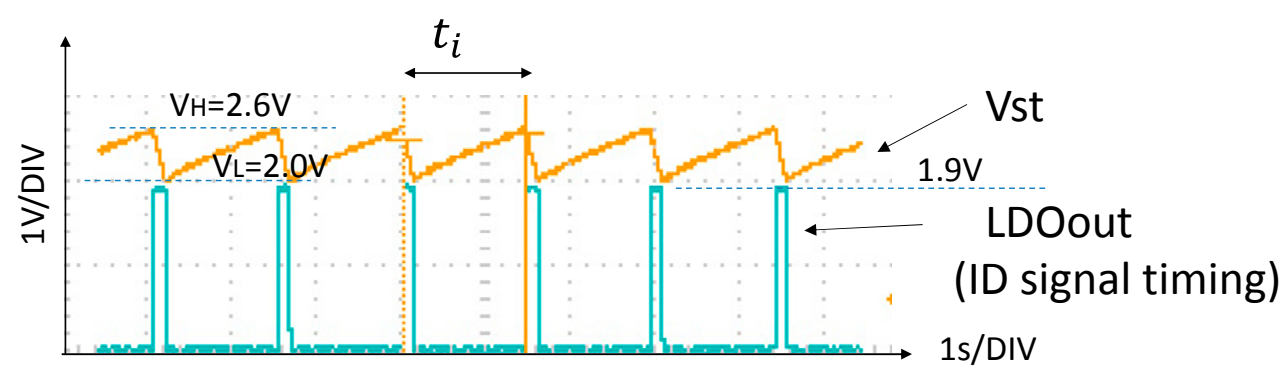

Figure 2. Vst and LDOout waveforms by DC Current Beat Sensors.

We used soft magnetic ferrite core clamp for L2 where coil for inductance is 11 turns. The inductance value is measured by LCR meter at a frequency of $100 \mathrm{KHz}$ as shown in Figure 3. The maximum inductance value is $147 \mathrm{uH}$, and it decreases as DC current to be measured (Io) decreases, and little hysteresis was observed. Therefore, as the DC current increases in the circuit, the interval time $t i$ is shortened. The interval time of the beats depends on the DC current to be measured.

$$
\begin{gathered}
\mathrm{E}_{1}=\frac{1}{2} \cdot \mathrm{L}_{1} \cdot \mathrm{i}_{1}{ }^{2} \\
\mathrm{E}_{1}=\frac{1}{2} \cdot \mathrm{L}_{2} \cdot \mathrm{i}_{2}{ }^{2}+\frac{1}{2} \cdot \mathrm{C}\left\{\mathrm{VST}^{2}(\mathrm{n})-\mathrm{VST}^{2}(\mathrm{n}-1)\right\}
\end{gathered}
$$
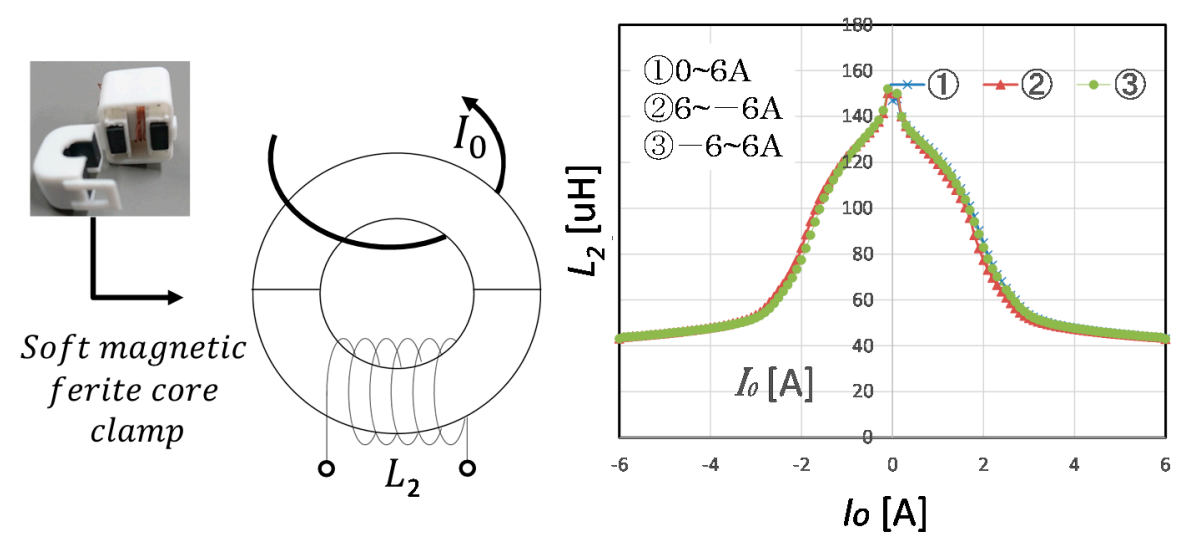

Figure 3. Measured inductance of the soft magnetic ferrite core clamp. The inductance depends on the DC Current which flows through the core. 
The photos of the measurement are shown in Figure 4. $\mathrm{L}_{1}$ inductance and $\mathrm{L}_{2}$ inductance are respectively made with coils around two cores of the two clamps. $\mathrm{L}_{1}$ of $18 \mathrm{uH}$, and $\mathrm{L}_{2}$ of $147 \mathrm{uH}$ were respectively obtained at the Io of $0 \mathrm{~A}$. The inductance of L2 depends on the DC current to be measured. A 2.4 GHz RF module (Nordic nPF24L01) with a output power of $0 \mathrm{dBm}$ [4] is used to transmit the ID code for the DCC beats. Measured communication range in line of sight was up to $50 \mathrm{~m}$ for the RF module. The switching frequency was $100 \mathrm{KHz}$. We have successfully obtained ID signals, the interval time of which corresponds to the DC current to be measured as shown in Figure 5. The power consumption of the circuit to generate the ID signals is typically $1.2 \mathrm{~mW}$ at the switching frequency of $100 \mathrm{KHz}$.

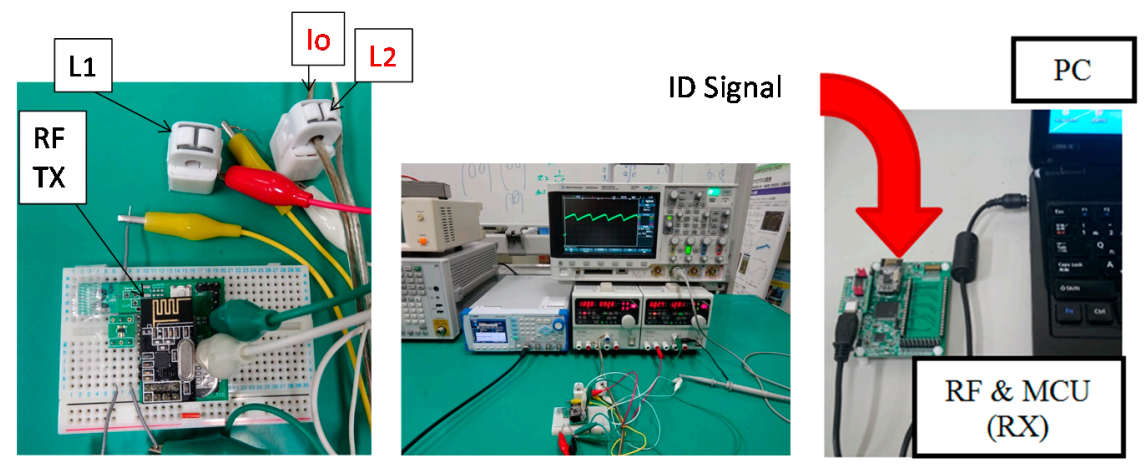

Figure 4. Measurement set up of the DCC Beat Sensor.

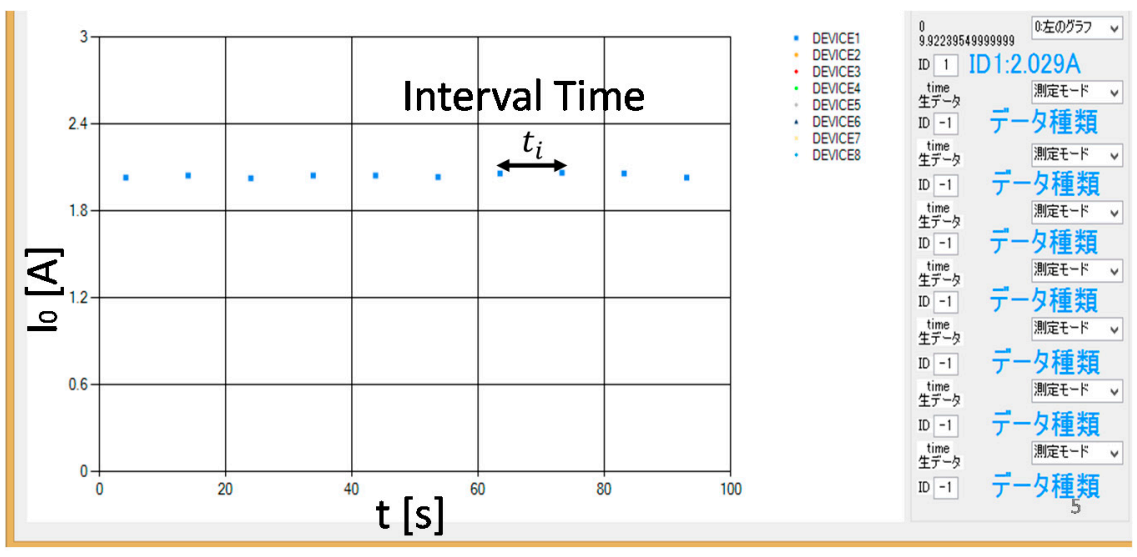

Figure 5. Measured DC current by the DCC Beat Sensor.

As shown in Figure 6a, the Io and $t i$ is correlated by the 4th order of correlation formula for calibration. Measured DC current obtained by the interval time corresponds to Io, and the error is max $5.7 \%$ at DC current of $0.2 \mathrm{~A}$ after calibration as shown in Figure $6 \mathrm{~b}$.

The specifications of the DCC Beat sensors are summarized and compared to commercially available hall type DC current clamp sensor as indicated in Table 1.

Table 1. Comparison Table of the DC current sensor

\begin{tabular}{ccc}
\hline & This Work & Hall Sensor (LAD-240) (1) \\
\hline Wired/Wireless & Wireless, up to $50 \mathrm{~m}$ comm. range & Wired \\
Non invasive & Yes & Yes \\
DC current range & 0.2 to $4 \mathrm{~A}$ & 1 to $200 \mathrm{~A}$ \\
Error & $5.7 \%$ after calibration & $2.5 \%$ \\
Resolution & $0.1 \mathrm{~A}$ & $1 \mathrm{~A}$ \\
Size & $40 \times 50 \times 20(\mathrm{TX}), 19 \times 19 \times 25 \mathrm{~mm}($ Clamp) & $44 \times 148 \times 21 \mathrm{~mm}$ \\
Consuming Power & $1.2 \mathrm{~mW}$ & Coin Battery LR-44 $\times 2$ \\
\hline
\end{tabular}



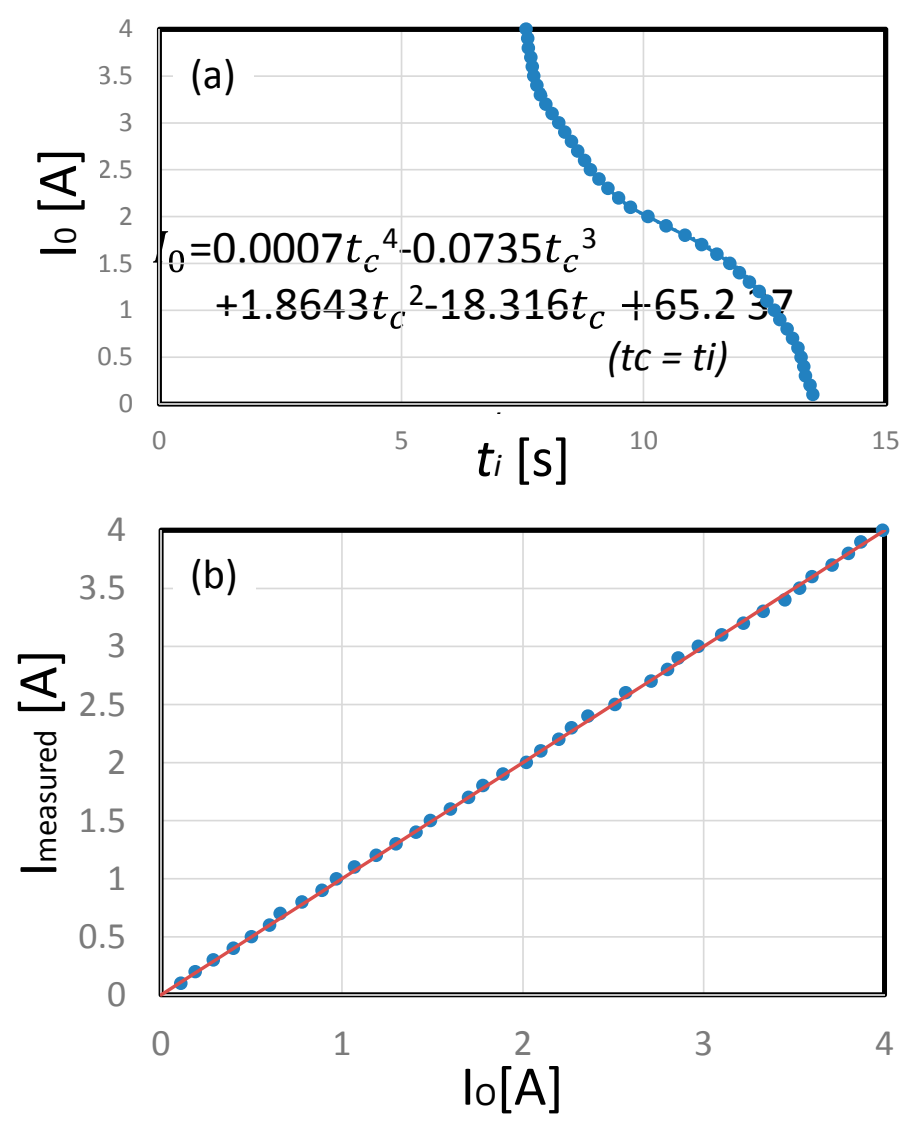

Figure 6. (a) Calibration formula for the DCC Beat Sensors; (b) Measured Io by the DCC beat sensors after calibration.

Conflicts of Interest: The authors declare no conflicts of interest.

\section{References}

1. Available online: http://www.multimic.com/products/detail/8 (accessed on 03/08/2017).

2. Ishigaki, S.; Ishibashi, K. Power Beat: A Low-cost and Energy Harvesting Wireless Electric Power Sensing Scheme for BEMS. In Proceedings of the 2015 IEEE International Conference on Building Efficiency and Sustainable Technologies, Singapore, 31 August-1 September 2015.

3. Takitoge, R.; Ishigaki, S.; Ishige, T.; Ishibashi, K. Temperature Beat: Persistent and Energy Harvesting Wireless Temperature Sensing Scheme. In Proceedings of the IEEE Sensors 2016, Orlando, FL, USA, 30 October-3 November 2016.

4. Available online: http://nordicsemi.com/eng/Products/2.4GHz-RF/nRF24L01 (accessed on 03/08/2017). 